\title{
Advances in Acute Myeloid Leukemia Management: Focus on Secondary Disease and Older Patients
}

\author{
Presented by Daniel A. Pollyea, MD, MS
}

\section{ABSTRACT}

The "Age of Induction" led to breakthroughs in the treatment landscape for acute myeloid leukemia (AML), and was immediately followed by a long period during which few drugs were approved. That all changed a few years ago, when 2017 began the "Age of Abundance." With many treatment options now available, new management strategies have emerged for patients with secondary AML, as well as for older patients with AML. Treatment can now be tailored to these special populations, and providers should be aware of the unique supportive care considerations associated with these newer AML therapies.

After several stagnant decades, the tides have shifted for the field of acute myeloid leukemia (AML). In the past several years, multiple new therapies have been approved, leaving AML providers with a multitude of new therapies. These advancements in the treatment of AML have also allowed for more treatment options for special populations, including patients with secondary AML and older patients.

At the NCCN 2020 Virtual Congress: Hematologic Malignancies, Daniel A. Pollyea, MD, MS, Associate Professor, Clinical Director of Leukemia Services, University of Colorado Cancer Center, took a dive into the treatment history of AML, provided a summary of the new treatment recommendations for secondary AML and AML in older patients, and reviewed the unique supportive care strategies that should be used in these populations.

\section{Treatment Strategies for Newly Diagnosed AML}

"Knowing about the history of drug development in AML goes a long way to understanding why we have the therapies we do and how we should use them," said Dr. Pollyea. First, 1973 ushered in the "Age of Induction," and introduced treatment with the $7+3$ regimen, a continuous infusion of cytarabine and intermittent dosing of an anthracycline. "Thousands of people all over the world have now been treated with this regimen, but unfortunately this was followed by a long second period that I call the 'Fallow Period,'” he said.

General incremental improvements over this era were attributable to advances in transplant or supportive care methods. "But in terms of new leukemia therapies that targeted the underlying disease, we saw very few, if any, developments during this period," he added. During this time when virtually no drugs were approved, multiple AML clinical trials were underway. "There were arguably more clinical trials for AML than for any other cancer during this period, but because we had such a limited understanding of the biological nature of this disease, we weren't able to treat it effectively," he said.

The third era was ushered in with a paper published by The Cancer Genome Atlas group in 2009. ${ }^{1}$ This group performed the first whole-genome sequencing of a patient with AML, resulting in a plethora of information. “And very quickly we've entered this era where it's standard of care to sequence and to understand every potential mutation that makes up an individual patient's disease," Dr. Pollyea said. "So, in a relatively short period of time, we made a massive leap forward in understanding the disease from a meta standpoint, but also on an individual level."

\section{Era of Abundance}

Beginning in 2017, the "Era of Abundance" was ushered in when multiple new therapies began to be approved, in the context of understanding the disease better than ever before. The latest drug for AML, CC-486, was approved just this year, and according to Dr. Pollyea, this period of abundance seems to be continuing.

A few important lessons should be gleaned from learning about the history of AML treatment, he noted. The first is that AML providers have been desperate for treatments. "That's sort of imprinted on our DNA because of that fallow period, and because of the difficulty and frustration of treating such a challenging disease with so few therapies," he said. Second, evaluating "fitness" for induction chemotherapy has endured even when it is no longer a binary choice between induction and no treatment. And finally, 
genomically targeted therapies continue to entice providers who treat AML, "perhaps to a fault," he suggested.

Dr. Pollyea illustrated the "conventional approach" to newly diagnosed AML and the pitfalls involved in such an approach (Figure 1). The first pitfall, he said, is that the field of AML currently has no standardized method to make a critical assessment of patient "fitness" for intensive chemotherapy. Other pitfalls to this approach include a lack of consensus regarding whether gemtuzumab can be used with induction chemotherapy for patients with intermediate-risk disease, whether CPX-351 should be avoided in patients with TP53 mutations and whether it should be given with an FLT3 inhibitor in the setting of FLT3 mutations, and whether induction chemotherapy and midostaurin should be used in older patients with FLT3 mutations. Finally, if a patient is deemed a good candidate for induction chemotherapy, does that necessarily mean it's the best treatment for that patient?

\section{Defining Secondary and Older AML}

Secondary AML could result from an antecedent hematologic disorder, most commonly myelodysplastic syndromes or a myeloproliferative neoplasm, or in rarer cases, a nonmalignant antecedent disorder (eg, aplastic anemia). ${ }^{2,3}$ Treatment-related AML is defined by the WHO as AML in a patient with a history of exposure to alkylating agents and/or topoisomerase II inhibitors and/or ionizing radiation to large fields including the bone marrow. ${ }^{3}$ Other implicated therapies include antimetabolites or antitubulin agents, but their relationship is less certain.

"Older AML is easier to define," he said. "Because AML is older AML. The median age at diagnosis is 68 years, so when we're talking about AML, we're talking about older patients."
Treatment Considerations for Secondary AML Liposomal daunorubicin and cytarabine (CPX-351) is a liposomal formulation of $7+3$. The original drug development led to no statistical improvement in overall survival compared with $7+3$ for all-comers in a study of patients with AML, but led to a statistically significant improvement in an unplanned secondary AML subgroup. ${ }^{4}$ This led to a confirmatory study for $7+3$ versus CPX-351 in patients with secondary AML, in which overall survival was improved for those who received CPX-351 versus $7+3$, especially those who went on to transplantation. 5 "So consider CPX-351 for patients with newly diagnosed, induction-eligible secondary AML, particularly if they would be suitable candidates for a transplant," Dr. Pollyea said.

In determining whether a patient has secondary AML, new research suggests that waiting up to 15 days for cytogenetic and molecular findings, if necessary, will not result in any worsening of outcomes. ${ }^{6}$ Other treatment options for this population, particularly for patients who are not eligible for induction chemotherapy, include glasdegib + low-dose cytarabine $^{7}$ or ivosidenib, which is applicable for patients with $I D H 1$ mutations $^{8}$ (present in $5 \%-10 \%$ of patients with AML). However, observed responses to these treatments in clinical trials have not been stratified by de novo versus secondary AML status. Similar data have been observed for enasidenib in newly diagnosed patients with $I D H 2$ positive disease, although there is currently no FDA label for this indication. "So we think there's a possibility that IDH inhibitors may have greater efficacy in combination with hypomethylating agents in the first-line setting, and we hope to see that data soon," he noted.

Another nonintensive therapy for patients with secondary AML is venetoclax + azacitidine. A recently

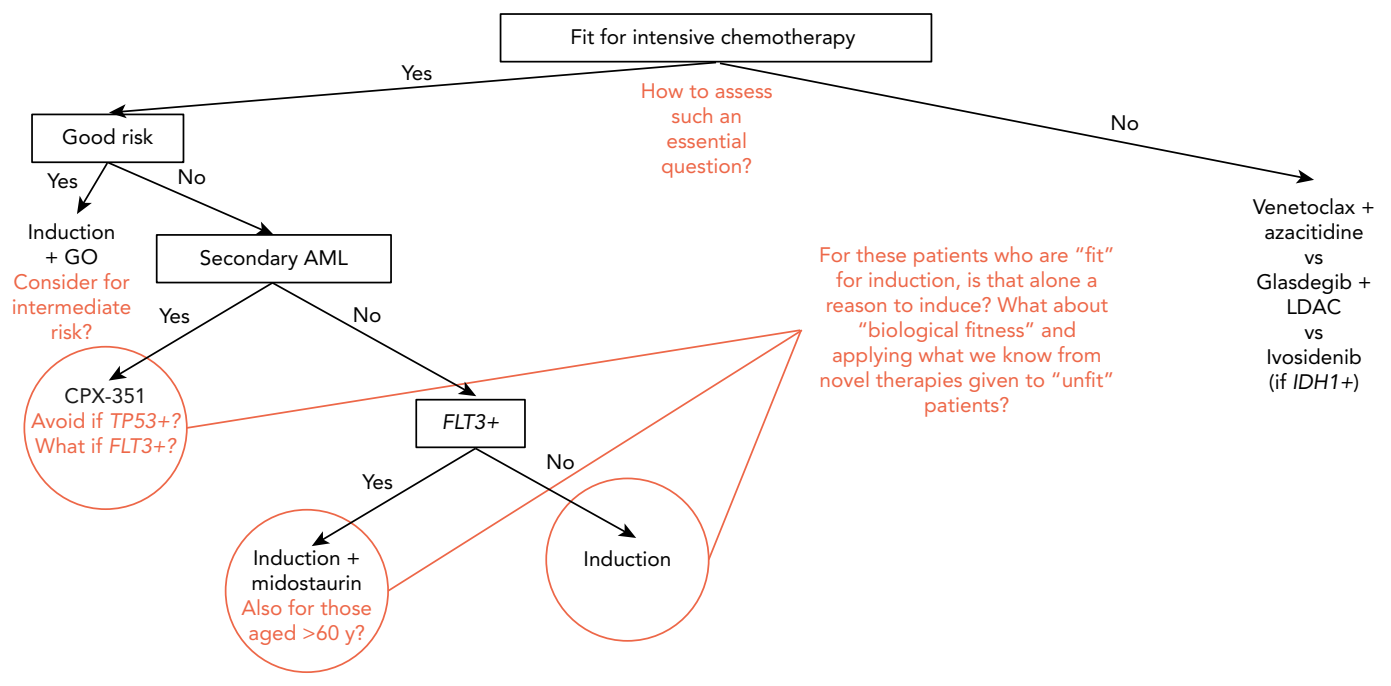

Figure 1. Conventional approach to newly diagnosed $A M L$ and pitfalls.

Abbreviations: AML, acute myeloid leukemia; GO, gemtuzumab ozogamicin; LDAC, low-dose cytarabine. 
published study in New England Journal of Medicine showed that patients with secondary AML and those with de novo AML demonstrated similar responses to the drug combination with respect to survival. ${ }^{10}$ "There isn't a big difference in outcomes for patients on venetoclax with secondary AML versus those with de novo AML," he said. "And that's a departure from expectations related to conventional treatments like chemotherapy, where patients with secondary AML traditionally do more poorly."

\section{Treatment Considerations for Older Patients}

There are inherent challenges to assessing "fitness" for intensive induction chemotherapy in older populations, but according to Dr. Pollyea, the typical recommendation is to lean toward intensive chemotherapy for patients with good-risk features, while acknowledging that this is rare. ${ }^{11}$ However, a real-world analysis of $>6,000$ patients with newly diagnosed AML demonstrated that this is not a particularly successful strategy in older patients, with upwards of $25 \%$ of patients aged 65 to 89 years being discharged to hospice or dying in the hospital. ${ }^{12}$ This is not only due to age, but also to worse disease biology that is less responsive to induction chemotherapy.

In older patients unfit for intensive chemotherapy, responses favor venetoclax/azacitidine. ${ }^{13}$ "So perhaps in the context of a venetoclax-based regimen, poor-risk cytogenetics don't have the bad predictive value they have for intensive chemotherapy," he suggested.

When it comes to prescribing treatment for older patients with AML, "I think that regardless of whether a patient is 'fit' for induction chemotherapy, the reality is that some of these therapies designed for 'unfit' patients may be better for the 'fit' patients," he said. "More work needs to be done here, but I think this is an unsettled area in the field."

\section{Supportive Care for Novel Therapies}

According to Dr. Pollyea, any patient receiving CPX-351 must also be able to tolerate intensive induction chemotherapy. Ongoing research is evaluating whether CPX-351 can be given on an outpatient basis as induction or consolidation therapy. When giving glasdegib, he warned of the potential for alopecia, QT prolongation, dysgeusia (consider dose interruption if severe), and muscle spasms (treat with hydration and antispasmodics).

IDH inhibitors (ivosidenib and enasidenib) can lead to differentiation syndrome in approximately $10 \%$ of patients. Treating this toxicity requires early recognition and steroid administration when suspected. All other causes should also be considered, because other conditions can mimic this toxicity in very sick patients with AML, and it is not recommended to hold drug except in very severe cases.

In rare cases, venetoclax can lead to tumor lysis syndrome, and therefore early recognition and supportive care are crucial. Providers should be aware of concomitant medications (dose-reduce venetoclax if using azoles or other CYP3A4 inhibitors), as well as the risk of cytopenias. "It's important that patients have a bone marrow biopsy at the end of cycle 1 to assess whether a prolonged cytopenia is due to refractory disease or the treatment itself," he advised. "And in patients experiencing response, it's very important to hold therapy for a period of time to allow recovery of blood counts between cycles."

Disclosures: Dr. Pollyea has disclosed that he is a scientific advisor for AbbVie, Inc., Amgen Inc., Bristol-Myers Squibb Company, Celgene Corporation, Genentech, Inc., GlycoMimetics, Inc., Karyopharm Therapeutics, Kiadis Pharma, Novartis Pharmaceuticals Corporation, Syndax Pharmaceuticals Inc., Syros Pharmaceuticals, and Takeda Pharmaceuticals North America, Inc.

Correspondence: Daniel A. Pollyea, MD, MS, University of Colorado Cancer Center, 1665 Aurora Court, Mail Stop F704, Aurora, CO 80045 Email: daniel.pollyea@ucdenver.edu

\section{References}

1. Mardis ER, Ding L, Dooling DJ, et al. Recurring mutations found by sequencing an acute myeloid leukemia genome. N Engl J Med 2009;361:1058-1066.

2. Greenberg $P$, Cox $C$, LeBeau MM, et al. International scoring system for evaluating prognosis in myelodysplastic syndromes. Blood 1997;89:2079-2088.

3. Arber DA, Orazi A, Hasserjian R, et al. The 2016 revision to the World Health Organization classification of myeloid neoplasms and acute leukemia. Blood 2016;127:2391-2405.

4. Lancet JE, Cortes JE, Hogge DE, et al. Phase 2 trial of CPX-351, a fixed 5:1 molar ratio of cytarabine/daunorubicin, vs cytarabine/daunorubicin in older adults with untreated AML. Blood 2014;123:3239-3246.

5. Lancet JE, Uy GL, Cortes JE, et al. CPX-351 (cytarabine and daunorubicin) liposome for injection versus conventional cytarabine plus daunorubicin in older patients with newly diagnosed secondary acute myeloid leukemia. J Clin Oncol 2018;36:2684-2692.

6. Rollig C, Kramer M, Schliemann C, et al. Does time from diagnosis to treatment affect the prognosis of patients with newly diagnosed acute myeloid leukemia? Blood 2020;136:823-830.

7. Cortes JE, Heidel FH, Hellmann A, et al. Randomized comparison of low dose cytarabine with or without glasdegib in patients with newly diagnosed acute myeloid leukemia or high-risk myelodysplastic syndrome. Leukemia 2019;33:379-389.

8. Roboz GJ, DiNardo CD, Stein EM, et al. Ivosidenib induces deep durable remissions in patients with newly diagnosed IDH1-mutant acute myeloid leukemia. Blood 2020;135:463-471.

9. Pollyea DA, Tallman MS, de Botton S, et al. Enasidenib, an inhibitor of mutant IDH2 proteins, induces durable remissions in older patients with newly diagnosed acute myeloid leukemia. Leukemia 2019;33:2575-2584.

10. DiNardo CD, Jonas BA, Pullarkat V, et al. Azacitidine and venetoclax in previously untreated acute myeloid leukemia. N Engl J Med 2020;383:617-629.

11. Leith CP, Kopecky KJ, Godwin J, et al. Acute myeloid leukemia in the elderly: assessment of multidrug resistance (MDR1) and cytogenetics distinguishes biologic subgroups with remarkably distinct responses to standard chemotherapy. A Southwest Oncology Group study. Blood 1997;89:3323-3329.

12. Zeidan $A M$, Wang $R$, Wang $X$, et al. Clinical outcomes of older patients with AML receiving hypomethylating agents: a large population-based study in the United States. Blood Adv 2020;4:2192-2201.

13. DiNardo CD, Jonas BA, Pullarkat $V$, et al. Azacitidine and venetoclax in previously untreated acute myeloid leukemia. N Engl J Med 2020;383:617-629. 